\title{
Traduire
}

Revue française de la traduction

$228 \mid 2013$

Technique et pragmatisme

\section{Traduire des normes}

Jacques Assael, Céline Bodo, Patricia Cohen, Pierrick Jeancolas et Annie Lestrade

\section{Q OpenEdition \\ Journals}

Édition électronique

URL : http://journals.openedition.org/traduire/526

DOI : $10.4000 /$ traduire.526

ISSN : 2272-9992

Éditeur

Société française des traducteurs

Édition imprimée

Date de publication : 1 juin 2013

Pagination : 38-48

ISSN : 0395-773X

Référence électronique

Jacques Assael, Céline Bodo, Patricia Cohen, Pierrick Jeancolas et Annie Lestrade, «Traduire des normes », Traduire [En ligne], 228 | 2013, mis en ligne le 01 juin 2015, consulté le 30 avril 2019. URL http://journals.openedition.org/traduire/526 ; DOI : 10.4000/traduire.526 


\section{Traduire des normes}

Jacques Assael, Céline Bodo, Patricia Cohen, Pierrick Jeancolas, Annie Lestrade

\section{Historique de la normalisation}

Depuis toujours, l'homme a éprouvé la nécessité de rationaliser ses techniques et ses modes de fonctionnement. II y a quelque 5000 ans, les Sumériens inventaient la plus ancienne écriture connue, comptaient en base 60 et nous léguaient la mesure du temps en heures, minutes et secondes. Plus près de nous, les ingénieurs militaires de Philippe Auguste, roi de France de 1180 à 1223, mettaient en place des critères types d'architecture militaire, bien avant que Vauban ne ceinture la France de citadelles et de fortifications. Voici deux siècles, l'industrialisation et l'apparition du chemin de fer bouleversaient les modes de consommation et les techniques de commercialisation. Les découvertes scientifiques, médicales et pharmaceutiques, essentielles au progrès de l'humanité, exigeaient quant à elles l'élaboration de protocoles de plus en plus complexes pour assurer la sécurité des chercheurs, des praticiens, des utilisateurs et des patients.

Au fil des ans se créèrent, notamment aux États-Unis, différentes associations d'ingénieurs dont le but était d'assurer la diffusion des nouvelles technologies de l'époque - la science des métaux, l'électricité, la télégraphie - dès la fin du xixe siècle. C'est ainsi que l'American Institute of Electrical Engineers, aujourd'hui I'Institute of Electrical and Electronics Engineers (IEEE), à l'origine de la création de l'Organisation internationale de normalisation (ISO), fut fondé en 1884 par des ingénieurs, dont Thomas Edison et Alexander Graham Bell pour ne citer que les plus célèbres. La Commission électrotechnique internationale (CEI), présidée par Lord Kelvin, est officiellement créée à Londres, en 1906, à l'instigation de dix nations, dont les États-Unis et la France.

En France, l'Association française de normalisation, l'AFNOR, a été fondée en 1926. L'AFNOR a été habilitée à délivrer la marque de conformité aux normes françaises NF le 10 janvier 1939 et a été reconnue d'utilité publique par le décret du 5 mars 1943. La première norme homologuée de l'AFNOR, la norme NF E27-681 de juin 1939 intitulée "Cales obliques pour poutrelles IPN profilées en $\mathrm{U}$ ou en $\mathrm{T}$ n figure toujours à son catalogue. 
Au lendemain de la guerre, en 1946, 65 délégués de 25 pays se réunissent à Londres pour envisager l'avenir de la normalisation internationale au sens large, la CEl étant spécialisée dans l'électrotechnique. En 1947, I'Organisation internationale de normalisation (ISO) voit officiellement le jour et crée 67 comités techniques. En 1951 paraît la première des normes ISO (appelées à l'époque Recommandations ISO), I'ISO/R 1:1951, Température normale de référence des mesures industrielles de longueur. Cette norme, qui a fait l'objet de plusieurs révisions, existe toujours sous la référence ISO 1:2002.

II manquait, au niveau européen, un organisme central capable de répondre aux besoins spécifiques de l'Europe naissante. Le Comité européen de normalisation (CEN) fut donc créé le 23 mars 1961, à l'initiative des bureaux de normalisation de la France, de l'Allemagne et du Benelux. L'AFNOR fut chargée d'assurer son secrétariat pendant la phase de lancement. Le CEN s'est depuis élargi et intègre, à l'heure actuelle, les 27 États membres de l'Union européenne, trois États membres de l'Association européenne de libre-échange (Islande, Norvège et Suisse), l'ancienne République yougoslave de Macédoine, la Croatie et la Turquie. Tout comme l'ISO et la CEI, le CEN ne traite pas de la technologie électronique, qui relève de la responsabilité du CENELEC, le Comité européen de normalisation électrotechnique. Ces deux organismes travaillent néanmoins en étroite collaboration.

En juin 1991, l'ISO et le CEN ont signé l'Accord de Vienne qui vise principalement à éviter la duplication des travaux de normalisation.

\section{La normalisation aujourd'hui}

Le développement fulgurant des nouveaux outils d'information et de communication ainsi que la mondialisation sans cesse croissante des échanges de biens et de services requièrent de plus en plus d'interopérabilité. Le souci des acteurs politiques et socio-économiques d'aujourd'hui est de favoriser cette interopérabilité, tout en mettant à disposition des consommateurs des produits de qualité, respectueux de l'environnement, inoffensifs pour la santé et répondant à de strictes conditions de sécurité.

La normalisation ne peut donc être qu'internationale et qui dit " international " dit " multilingue ". La traduction s'avère désormais une étape primordiale dans la création et la mise à jour des normes.

Les domaines de traduction sont nombreux. Pour s'en convaincre, il suffit de consulter la liste des secteurs techniques de l'AFNOR :

Aéronautique et spatial, Agroalimentaire, Automobiles, Banques/Assurances/Finances, Biens de consommation/Sports et loisirs, Construction, Eau et assainissement, Énergie, Industrie, Santé et médico-social, Services, Technologies de l'information et de la communication, Tourisme, Transport et logistique. 
Chacun de ces secteurs techniques est lui-même divisé en domaines. Par exemple, l'agroalimentaire ne comporte pas moins de 24 domaines.

\section{Standardisation ou normalisation ?}

Voilà une question qui est souvent posée, d'autant qu'en anglais, par exemple, il n'existe pour couvrir ces deux vocables qu'un seul terme : standardisation. Certes, "standardiser " et " normaliser " sont des termes " voisins ", souvent perçus comme des synonymes et présentés comme tels dans bien des dictionnaires. Il existe toutefois une nuance entre ces deux mots en français.

Si la normalisation et la standardisation consistent en effet à définir des règles, des lignes directrices, des caractéristiques ou encore des performances communes pour des produits, des activités ou leurs résultats, elles n'ont pas la même portée.

L'AFNOR précise qu'une norme est approuvée par un organisme reconnu (AFNOR, CEN, ISO, etc.). Elle est généralement destinée à s'appliquer à tout un secteur, tandis qu'un standard est fondé sur un consensus plus restreint : il est généralement élaboré par une entreprise ou un consortium d'industriels.

\section{Fonctionnement du système de normalisation}

Le Système français de normalisation, tel que nous le connaissons aujourd'hui, est régi par le décret $n^{\circ} 2009-697$ du 16 juin 2009.

La normalisation et sa promotion sont assurées par l'AFNOR, ainsi que par les organismes délégués par l'AFNOR et agréés comme Bureaux de normalisation sectoriels par le ministre chargé de l'industrie. II existe actuellement 25 bureaux de normalisation sectoriels. L'AFNOR, en tant qu'animateur central de la normalisation en France, recense les besoins en normalisation et mobilise les parties intéressées. Elle promeut les positions françaises aux niveaux européen et international. Elle organise une consultation publique en français sur chaque projet de norme française, européenne ou internationale, homologue la version finale et l'intègre au catalogue national. Elle assure un travail de veille pour garantir que les normes publiées restent pertinentes.

L'AFNOR publie des normes nationales, d'application volontaire ou obligatoire. Les normes dont l'application est rendue obligatoire par arrêté signé du ministre concerné sont consultables gratuitement sur le site de l'AFNOR.

Le Comité européen de normalisation publie des normes dites " normes européennes " ou " normes EN ", d'application volontaire ou obligatoire. Ces normes sont destinées à favoriser les échanges, la concurrence, la sécurité des travailleurs et des consommateurs, l'interopérabilité des réseaux de transport, d'énergie, etc., au niveau européen. 
Les normes obligatoires, dites " harmonisées ", qui représentent environ $30 \%$ des normes du CEN, permettent la mise en pratique des directives européennes appelées " directives nouvelle approche ". Ces directives européennes s'accompagnent d'un mandat de normalisation délivré au CEN par la Commission européenne. Les normes harmonisées sont citées au Journal officiel de l'Union européenne et leur application est obligatoire pour tous les pays membres du CEN qui doivent retirer de leur catalogue les normes nationales en contradiction.

L'ISO publie des normes d'application volontaire, dont l'adoption représente pour les entreprises un outil stratégique important, ouvrant l'accès à de nouveaux marchés. La famille de normes ISO 9000 - Systèmes de management de la qualité, I'ISO 26000 - Responsabilité sociétale et l'ISO 31000 - Management du risque, figurent parmi les normes les plus connues.

Toutes les normes sont revues au bout de cinq ans, afin de s'assurer de leur pertinence et de la nécessité ou non d'une mise à jour. Les normes naissent, vivent, meurent ou renaissent sous une autre forme.

L'origine des normes est facilement identifiable, dans le catalogue de l'AFNOR, grâce à un système de lettres de référence :

NF indique l'origine française, EN l'origine européenne et ISO l'origine internationale. C'est ainsi que :

- une norme NF est une norme entièrement française ;

- une norme NF ISO est une norme internationale reprise en France ;

- une norme NF EN est une norme européenne reprise en France ;

- une norme NF EN ISO est une norme d'origine internationale reprise en Europe et en France.

Au niveau national, tout acteur socio-économique français peut proposer un projet de norme. Si la proposition est retenue, les partenaires intéressés se réunissent au sein d'une commission. La proposition peut être traitée au niveau national, européen ou mondial.

Au niveau européen et au niveau international, la demande de proposition de travaux de normalisation doit être formulée par l'organisme national de normalisation membre du CEN ou de l'ISO, en l'occurrence l'AFNOR pour la France.

L'élaboration d'une norme, qu'elle soit nationale, européenne ou internationale, repose sur le principe du consensus entre l'ensemble des parties prenantes d'un marché ou d'un secteur d'activité et, pour l'essentiel, comporte les étapes suivantes :

- l'étude de la pertinence de la demande de norme ;

- la création d'un groupe de travail regroupant toutes les parties concernées ;

- la recherche d'un document éventuel déjà existant sur lequel s'appuyer ;

- la rédaction du projet de norme ; 
- une période consacrée à l'enquête nationale ouverte à tous et à la collecte des commentaires et avis des pays membres ;

- la rédaction du texte final ;

- un vote formel ;

- la publication.

L'élaboration d'une norme peut nécessiter entre une et trois années de travail.

\section{L'aspect linguistique}

II va sans dire que, dans un cadre ayant une dimension internationale aussi marquée, l'aspect linguistique va prendre toute son importance. Au niveau du CEN, par exemple, un projet de norme, initialement rédigé soit en allemand, soit en anglais, soit en français, doit être disponible dans ces trois langues pour les besoins des enquêtes préliminaires. La traduction doit également faire l'objet d'une révision au moment de sa publication pour tenir compte des modifications apportées au texte originel pendant le processus de validation.

De la même manière, au niveau de l'ISO, un projet de norme rédigé en anglais doit être disponible en français au moment des enquêtes préliminaires et pour sa publication.

En France, comme dans d'autres pays, c'est dans ce cadre qu'intervient le traducteur dans le processus d'élaboration des normes CEN et ISO, soit pour le compte de l'organisme national de normalisation (AFNOR dans le cas présent) soit pour les bureaux de normalisation sectoriels.

\section{Annie, Céline, Patricia, Jacques et Pierrick : qui sommes-nous ?}

Pour répondre aux appels d'offres des organismes de normalisation, notre réseau devait regrouper des traducteurs possédant non seulement des compétences évidentes en traduction, mais également des compétences techniques reconnues.

Nous sommes parvenus à un équilibre entre traducteurs de formation et traducteurs autodidactes de manière toute naturelle : Annie, assistante de direction export et Jacques, ingénieur en électronique, après avoir longtemps travaillé respectivement dans l'industrie et la recherche, traduisaient des brevets. Patricia avait opté également pour la traduction de brevets après avoir obtenu un baccalauréat scientifique et suivi un cursus universitaire en traduction spécialisée. Suite aux difficultés rencontrées par les traducteurs de brevets lors de la signature du Protocole de Londres, tous trois ont souhaité réorienter leur activité vers la traduction de normes. Céline s'est formée à la traduction technique dans le cadre d'un DESS en alternance à Paris-Diderot. Elle a d'ailleurs effectué son apprentissage au sein du Pôle Traduction de 
I'AFNOR. Elle a ensuite travaillé comme chef de projet dans une agence de traduction. Pierrick, titulaire d'un baccalauréat scientifique, est venu tout naturellement à la traduction technique après des études universitaires en traduction.

Outre nos compétences en traduction, nous allions des compétences techniques ou scientifiques acquises soit par notre formation, soit par notre expérience, et que nous avons pris soin d'entretenir ou de développer.

\section{Comment travaillons-nous?}

Travailler en normalisation implique de devoir s'adapter à des volumes de travail irréguliers.

En effet, en fonction des réunions de la commission ou du comité technique concerné, il n'est pas rare que les traductions, pour un même domaine, arrivent par vagues. L'avantage indéniable de travailler en réseau nous permet de pouvoir œuvrer sur plusieurs traductions simultanées ou successives et d'en effectuer des révisions croisées. Ce mode de fonctionnement, associé à l'utilisation d'un outil de TA0, nous permet de préserver la cohérence terminologique nécessaire entre les documents traduits.

Une fois ces normes publiées, si la commission ou le comité technique estime qu'il n'est plus utile d'élaborer de normes supplémentaires, il s'écoulera une période de 3 à 5 ans avant que les normes soient reprises et qu'elles fassent l'objet d'une éventuelle révision.

Il est donc important de posséder des compétences suffisantes permettant de travailler dans plusieurs domaines.

S'il est des domaines techniques partagés par toute l'équipe, comme la sécurité et la santé au travail, l'ingénierie industrielle, les biens d'équipements et les matériaux, nous sommes parvenus à constituer des trinômes ou des binômes dans d'autres domaines : par exemple, Patricia et Pierrick travaillent en priorité sur des normes traitant de biologie et de chimie, Annie, Céline et Jacques traduisant des normes sur les machines et la mécanique.

En outre, chacun a ses sujets de prédilection, des passions qui lui sont propres : Annie traduit les normes de management et de gestion des informations, Céline privilégie les normes de santé et les énergies renouvelables, Patricia est la spécialiste de l'eau et des sols, Jacques est féru de télécommunications et de micro-électronique. Pierrick s'intéresse, lui aussi, aux énergies renouvelables et se passionne pour les nanotechnologies.

Notre entourage, amical, familial, professionnel, est souvent déconcerté par la passion que nous portons à nos travaux. II s'étonne que nous puissions prendre autant à cœur des sujets a priori ardus et dénués de poésie. En fait, au fur et à mesure de nos recherches terminologiques et techniques, de l'avancée de notre travail de traduction, nous voyons se dérouler devant nous des processus que nous pouvons matérialiser et, pour certains, personnaliser : 
il nous arrive de nous représenter le laborantin devant son microscope, le concepteur devant sa machine ou l'informaticien devant son clavier. Nous nous approprions " nos " normes, " nos " domaines, les objets de " nos " normes. Nous nous surprenons à prononcer des phrases qui ne manqueraient pas de provoquer l'hilarité du non-initié : " Pierrick a révisé les canalisations de Patricia ", " Annie a livré ses compteurs ", " Jacques a envoyé sa dameuse en validation " ou autres "Céline est sur ses implants "...

Il est néanmoins un piège que nous avons le souci d'éviter : la normalisation ayant sa terminologie et une syntaxe propres, nous nous sommes rendu compte que nous étions conditionnés par cette terminologie et cette syntaxe et que, si nous n'y prenions pas garde, nous risquions d'appauvrir notre capital linguistique. II est donc important qu'en dépit de toutes les satisfactions que nous retirons de notre travail, nous continuions à exercer nos compétences dans d'autres secteurs d'activité pour ne pas perdre la fluidité rédactionnelle indispensable à notre métier. Ainsi, Céline et Patricia traduisent depuis l'allemand et Pierrick depuis l'italien des documents non normatifs. Annie et Jacques ne travaillant que depuis l'anglais, Annie s'est spécialisée dans les communiqués de presse techniques et Jacques poursuit son activité dans les brevets.

\section{Le processus de traduction en normalisation}

Le traducteur ayant choisi de se spécialiser dans le domaine de la normalisation doit maîtriser ses différents thèmes de prédilection pour atteindre le niveau de qualité requis.

De surcroît, il va devoir se conformer à un cadre strict, car la présentation générale d'une norme est rigoureusement standardisée. Toute norme comporte :

- des mentions d'ordre administratif et juridique précédant un sommaire détaillé et un avantpropos;

- un domaine d'application stipulant avec précision ce que la norme englobe et n'englobe pas ;

- des références normatives spécifiant les autres documents normatifs auxquels la norme fait référence et que le lecteur doit consulter;

- une partie " termes et définitions ", précieuse pour comprendre la terminologie utilisée dans la norme ou dans la série de normes associées ;

- éventuellement des listes légendées de symboles et de sigles ;

- puis le corps du texte normatif proprement dit ;

- viennent ensuite des annexes " normatives " (dont les prescriptions doivent être appliquées dans le cadre d'une revendication de conformité à la norme) ou purement " informatives " ;

- enfin, la norme se termine par une bibliographie mentionnant les normes, les parutions scientifiques ou techniques, les directives européennes et autres documents ayant servi à son élaboration. 
II arrive que les " termes et définitions " fassent l'objet d'une norme à part entière ou de l'essentiel d'une norme. C'est le cas, par exemple de :

- la norme NF EN ISO 1942 - Médecine bucco-dentaire - Vocabulaire

- la norme NF EN 13551 - Bateaux de navigation intérieure - Vocabulaire

- la norme NF ISO 6289 - Skis - Vocabulaire

Rappelons que si certaines normes sont d'application volontaire, d'autres ont un caractère réglementaire. Les entreprises soucieuses de se démarquer de leurs concurrents ou d'atteindre le même niveau de qualité que ceux-ci s'engagent donc dans des programmes de certification.

Les normes techniques pouvant servir d'outils réglementaires, la terminologie revêt une importance particulière : il est ainsi primordial de faire la différence entre ce qui est conseillé et ce qui est formellement exigé, d'où l'utilisation systématique des modaux shall, traduit en français par " il faut "/ on doit ", et de should, traduit par " il convient de ". Le modal must est fort peu utilisé en normalisation. De même, il est fortement recommandé d'éviter l'utilisation du conditionnel en français.

Le traducteur doit préserver une cohérence terminologique tout au long de la norme en s'efforçant de restituer le message en phrases simples, quitte à devoir effectuer des répétitions. "Traduire une norme, ce n'est pas traduire Shakespeare, c'est penser à l'utilisateur final ». Tel fut le premier conseil délivré par la responsable du Pôle Langues de l'AFNOR, traductrice ellemême et donc consciente du souci de vouloir " bien faire " des traducteurs : en la matière, le mieux peut être l'ennemi du bien.

II va également sans dire que le processus de traduction doit respecter la procédure stipulée dans la NF EN 15038 (norme française d'origine européenne) Services de traduction Exigences requises pour la prestation du service. II serait, en effet, pour le moins étrange que des traducteurs spécialisés en normalisation ne respectent pas la norme qui s'applique à leur profession!

L'utilisation d'un outil de TA0 est également essentielle. Comment maintenir l'homogénéité des textes, se conformer à la virgule près aux versions précédentes, si l'on ne dispose pas d'une mémoire de traduction permettant de conserver soigneusement, sous un format "standard ", le vocabulaire et les expressions qui doivent s'employer de façon systématique?

Sur le plan technique, il est évident que le traducteur doit disposer de bonnes connaissances théoriques dans les domaines de spécialité qu'il aborde, non seulement celles acquises grâce à son expérience professionnelle, mais également celles entretenues par le biais de formations, de lecture d'ouvrages spécialisés, etc.

L'organisme de normalisation, le client donc, fournit, avec le document à traduire, les éventuelles versions antérieures ou des indications de normes à consulter. Le traducteur peut, bien 
entendu, demander à disposer de références supplémentaires. Ces documents lui permettent de se constituer un corpus de référence fiable et une terminologie cohérente et unique. En effet, il n'est pas rare qu'un même concept technique soit traduit de plusieurs manières différentes en fonction des fabricants ou des utilisateurs finaux. Les versions antérieures éventuellement disponibles servent dès lors à alimenter la création de cette mémoire de traduction si essentielle.

Lorsque la traduction est achevée, le traducteur en effectue une révision approfondie avant de la transmettre à sa consœur ou son confrère, le relecteur, qui effectue, de son côté, une révision supplémentaire. Le relecteur renvoie son travail au traducteur qui valide ou non ses corrections.

Ensuite, la traduction part chez un " expert valideur ", à savoir un membre de la commission technique de I'AFNOR spécialiste du domaine traduit, qui va effectuer une révision technique et terminologique.

Les rôles du traducteur et de l'expert valideur sont bien déterminés. Le traducteur, spécialiste de la langue, reste maître de la syntaxe ; l'expert valideur confirme ou spécifie la terminologie technique appropriée et valide l'interprétation faite des processus d'essais ou de fabrication. En effet, il arrive fréquemment que des normes rédigées en anglais par des techniciens dont ce n'est pas la langue maternelle soient relativement confuses. Seules l'expérience et les connaissances de l'expert valideur permettent de juger de la validité technique de la traduction.

L'expert valideur transmet à l'AFNOR une fiche d'appréciation sur le travail effectué par le traducteur et renvoie sa validation technique au traducteur qui intègre les corrections demandées ou contacte l'expert valideur en cas de désaccord ou pour obtenir des informations complémentaires.

Le traducteur procède ensuite à la livraison du document traduit à l'AFNOR ou au bureau sectoriel concerné.

Des contrôles qualité sont régulièrement effectués par l'AFNOR sur les documents traduits et des fiches d'évaluation sont communiquées à l'entreprise de traduction. Ces contrôles qualité permettent de confirmer les domaines de spécialité pour lesquels elle a répondu à un appel d'offres.

Certes, la traduction de normes peut être on ne peut plus irritante lorsqu'il faut jongler avec les outils informatiques, les formules, les symboles, la mise en page chaotique et la conversion capricieuse des documents. II est également frustrant pour un traducteur de devoir appliquer des contraintes stylistiques et syntaxiques et jouer à la devinette avec des documents mal rédigés. 
Néanmoins, elle est aussi source de plaisir : le plaisir de découvrir ou de suivre les évolutions techniques de nos domaines de spécialité, de se former et de s'informer en permanence par la relecture des travaux de nos confrères, d'effectuer des recherches approfondies sur des sites de professionnels, dans les documents de l'Union européenne, dans des bases de données techniques, de se confronter aux néologismes, voire aux barbarismes, pour tenter de trouver la solution la plus adaptée.

Il est également très valorisant pour un traducteur d'apporter sa pierre, fût-elle modeste, à un processus visant à améliorer la qualité et la sécurité des services et des produits commercialisés, de participer, à son échelle, à la protection de l'environnement et à l'amélioration des communications informatiques entre autres. C'est également une façon de participer à la vie économique.

En effet, la possibilité d'influer sur les règles et normes internationales est une composante essentielle de la compétitivité des entreprises et des États. Les normes représentent des enjeux économiques majeurs et font l'objet d'une concurrence rude entre les pays pour imposer leurs standards. La prise de position récente de la Commission européenne en faveur des standards allemands pour les prises des véhicules électriques en est la dernière illustration.

Madame Nicole Bricq, ministre du Commerce extérieur, pour qui « la bataille économique, c'est aussi la bataille normative ", a commandé en septembre 2012 un rapport à Madame Claude Revel, conseillère du Commerce extérieur de la France. Voici, pour conclure, quelques extraits du résumé exécutif du rapport :

L'influence normative : pourquoi?

L'influence sur les règles et normes internationales, c'est-à-dire sur les règles du jeu économique, est une composante essentielle, quoique peu visible, de la compétitivité des entreprises et des États. Les régulations internationales ne sont jamais innocentes, elles déterminent des marchés, fixent des modes de gouvernance, permettent à leurs auteurs de devancer la concurrence, ou de la freiner, ou d'exporter leurs contraintes.

[...] En même temps, la norme comme la règle ont toujours pour objet de sécuriser les échanges, de faciliter l'interopérabilité du commerce et, en principe, de protéger le consommateur tout en lui assurant le meilleur service. De ce point de vue, le rapport suggère de proposer une meilleure prise en compte de la traçabilité et de la durabilité dans la norme. 


\section{Quelques liens utiles}

Site de l'AFNOR : www.afnor.org/

Site de l'AFNOR consacré aux enquêtes publiques : www.enquetes-publiques.afnor.org

Site de la CEl : www.iec.ch/

Site du CEN : www.cenorm.be

Site du CENELEC : www.cenelec.eu/ -

Site de l'IEEE : http://www.ieeefrance.org/

Site de l'ISO : http://www.iso.org/iso/fr

Le rapport de Mme Claude Revel est téléchargeable à l'adresse :

http://www.commerce-exterieur.gouv.fr/renforcer-influence-france-sur-normes-internationalesrapport-revel

Jacques Assael, Céline Bodo, Patricia Cohen, Pierrick Jeancolas, Annie Lestrade sont cinq traducteurs, qui ont constitué un réseau pour répondre aux appels d'offres publics et privés en matière de normalisation. Ils sont unis par les mêmes objectifs, un esprit d'équipe, un souci de solidarité et des complémentarités professionnelles, techniques et scientifiques qui leur permettent de traduire dans divers domaines techniques et scientifiques, et pour plusieurs bureaux de normalisation.

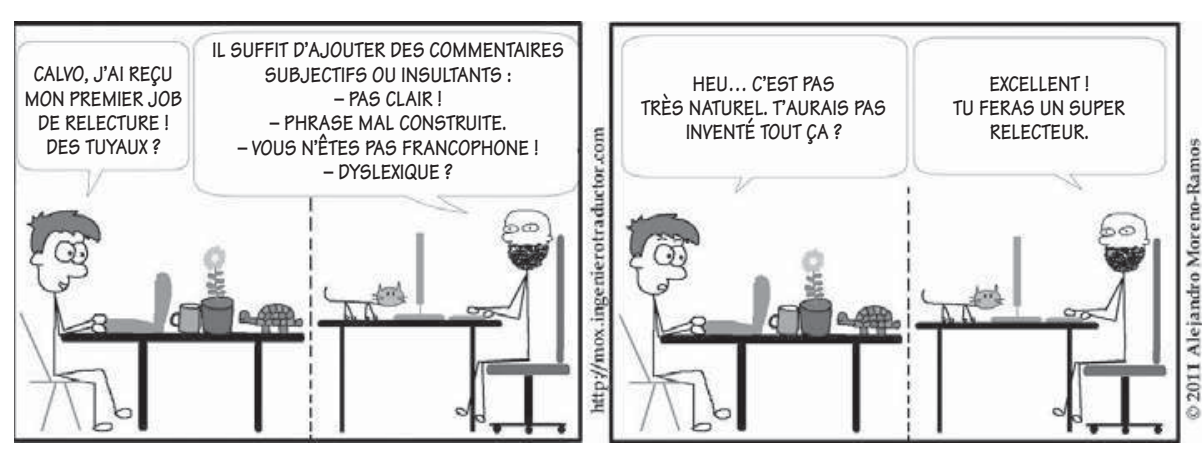

Relecteur, mode d'emploi 